\title{
Frequency domain interferometry mode observations of PMSE using the EISCAT VHF radar
}

\author{
P. B. Chilson ${ }^{1, *}$, S. Kirkwood ${ }^{1}$, I. Häggström ${ }^{2}$ \\ ${ }^{1}$ MRI Atmospheric Research Programme, Swedish Institute of Space Physics, Box 812, S-981 28 Kiruna, Sweden \\ 2 Swedish Institute of Space Physics, Box 812, S-981 28 Kiruna, Sweden \\ * Current affiliation: CIRES, University of Colorado/NOAA and NOAA Environmental Technology Laboratory, 325 Broadway, \\ R/ET2, Boulder, CO, 80305-3328, USA e-mail: Phillip.Chilson@noaa.gov
}

Received: 21 February 2000 / Revised: 2 October 2000 / Accepted: 11 October 2000

\begin{abstract}
During the summer of 1997 investigations into the nature of polar mesosphere summer echoes (PMSE) were conducted using the European incoherent scatter (EISCAT) VHF radar in Norway. The radar was operated in a frequency domain interferometry (FDI) mode over a period of two weeks to study the frequency coherence of the returned radar signals. The operating frequencies of the radar were 224.0 and $224.6 \mathrm{MHz}$. We present the first results from the experiment by discussing two 4-h intervals of data collected over two consecutive nights. During the first of the two days an enhancement of the FDI coherence, which indicates the presence of distinct scattering layers, was found to follow the lower boundary of the PMSE. Indeed, it is not unusual to observe that the coherence values are peaked around the heights corresponding to both the lower- and upper-most boundaries of the PMSE layer and sublayers. A Kelvin-Helmholtz mechanism is offered as one possible explanation for the layering structure. Additionally, our analysis using range-timepseudocolor plots of signal-to-noise ratios, spectrograms of Doppler velocity, and estimates of the positions of individual scattering layers is shown to be consistent with the proposition that upwardly propagating gravity waves can become steepened near the mesopause.
\end{abstract}

Key words: Ionosphere (polar ionosphere) Meteorology and Atmospheric Dynamics (middle atmosphere dynamics) - Radio Science (Interferometry)

\section{Introduction}

The mesosphere-stratosphere-troposphere (MST) class of Doppler VHF radars has been used for remote

Correspondence to: P. B. Chilson sensing of the atmosphere since the early 1970s. VHF radar signals are weakly backscattered from meter-scale irregularities of refractive index which, at mesospheric altitudes, are caused by fluctuations of electron density. The radar returns from these heights are particularly weak and are only detected from intermittently occurring layers of thin vertical extent. Ecklund and Balsley (1981) were the first to report the observation of abnormally strong and persistent radar returns from the mesopause region (around $85 \mathrm{~km}$ altitude) above Alaska during the mid-summer months. These echoes have been termed polar mesosphere summer echoes (PMSE), although they have also been observed at midlatitudes (Reid et al., 1989; Thomas et al., 1992; Chilson et al., 1997a). Traditional theories of radar (partial) reflection and scattering have been unable to explain the PMSE and the exact mechanism for their occurrence remains unclear despite the steadily increasing interest in them over the past 15 years.

Several intensive campaigns have complemented radar observations of PMSE with simultaneous lidar soundings and in-situ measurements of the mesopause region with rocket-borne probes. An overview of these campaigns is given in Lübken (1999). It has been established that the PMSE are in some way associated with the occurrence of noctilucent clouds (NLC), diffuse layers of ice crystals which constitute the highest observed clouds in our atmosphere (Nussbaumer et al., 1996; von Zahn and Bremer, 1999; Stebel et al., 2000). For example, the occurrence of both phenomena are confined to a narrow altitude region around $85 \mathrm{~km}$. They both follow a seasonal pattern of abrupt appearance in late May and a more gradual disappearance in mid-August (Kirkwood et al., 1998; Hoffmann et al., 1999). This period corresponds roughly to the time between the completion of the summertime cooling of the polar mesopause to the time of reversal of the mesospheric circulation to autumn conditions. In this connection, both phenomena are associated with the extremely low temperatures $(<140 \mathrm{~K})$, which are unique to the polar summer mesopause. Currently accepted theories regarding the mechanism giving rise to PMSE 
agree that one of the conditions needed for enhanced radar echoes is the presence of low-mobility charge carriers such as large cluster ions or macroscopic "dust" particles which capture the ambient electrons.

PMSE cannot be explained by traditional radar scatter theories, since the turbulent fluctuations near the mesopause generally exist at scales outside of the inertial subrange for those frequencies used by MST radars. However, for large Schmidt numbers $\left(S_{c}=v / D\right.$, where $v$ is the neutral atmospheric molecular diffusion rate and $D$ is the electron diffusion rate), the inertial subrange of the plasma spectrum can be extended to sufficiently small scales to allow the Bragg condition to be fulfilled. This can be attributed to, e.g., low mobilities of charged aerosols or dust particles. Indeed, evidence for Schmidt numbers as high as 420 in connection with PMSE have been recently reported by Lübken et al. (1998).

Plasma fluctuations caused by neutral turbulence can account for some of the PMSE signatures that have been observed; however, Lübken et al. (1993) have convincingly shown that PMSE can exist at altitudes where no neutral turbulence is present. Therefore we must additionally consider mechanisms for generating PMSE that do not directly require turbulence. Further evidence of this can be found in those PMSE signals that are aspectsensitive. For radar backscatter to be aspect sensitive means that the echoes are strongest when the transmitted radio waves are incident at right angles to some spatially extended refractivity structure in the atmosphere. Hocking and Röttger (1997) state that the aspect sensitivity of the PMSE might be explained in terms of (a) sharp edges on turbulent layers, (b) highly anisotropic scatterers at the edges of turbulent layers, and/or (c) small-scale highly damped wave-like modes similar to viscosity waves.

For a detailed understanding of the radar return mechanisms, it appears necessary to employ interferometric methods with effective resolutions of the order of tens of meters. This represents at least an order of magnitude improvement over standard MST radar resolutions. For example, one of the theories proposed to explain the occurrence of PMSE is that of dust-hole scatter, attributed to Havnes et al. (1992). It is assumed that horizontal vortex rolls are embedded in a field of falling charged dust or ice aerosols. The core of the vortex is free of aerosols, which leads to a sharp gradient in the aerosol density at the boundaries. If the aerosols are charged, this in turn leads to electron and ion density gradients. If such vortices exist then it is expected that their structure could be probed by the interferometric techniques but that they could not be resolved by the conventional radar methods.

Additionally, interferometric observations of PMSE will contribute to our understanding of the fine-scale atmospheric dynamics of the polar summer mesopause region. In the absence of PMSE, it is difficult to obtain high temporal and spatial resolution radar wind data from this region due to weak echo power. Therefore, it is not surprising that several studies of atmospheric dynamics have been conducted in which PMSE are treated as tracers of the underlying wind fields.
We have made measurements using the EISCAT VHF Radar $\left(69.58^{\circ} \mathrm{N}, 19.22^{\circ} \mathrm{E}\right)$ in Troms, Norway to investigate PMSE. The objectives of the observations included both determining the morphology and smallscale vertical structures of echoes. To facilitate an investigation into the small-scale vertical structures the radar was operated in a frequency domain interferometry (FDI) mode. This allows detection of the presence of thin scattering layers within the radar volume. We note that FDI has proven effective in a number of studies in the lower to middle atmosphere (Kudeki and Stitt, 1990; Chilson et al., 1997b; Muschinski et al., 1999); however, with the exception of one 37-min data set of observations (Franke et al., 1992), this technique has not been utilized to date for the study of PMSE.

\section{Experiment}

The EISCAT VHF radar has a nominal frequency of $224 \mathrm{MHz}$, but is capable of operating at frequencies ranging from $222.4-225.4 \mathrm{MHz}$ in steps of $200 \mathrm{kHz}$. During this experiment the radar was operated in a twofrequency FDI mode at 224.0 and $224.6 \mathrm{MHz}$. That is, the radar transmitted and received on two alternating frequencies as opposed to the typical arrangement in which only one frequency is used. We discuss the FDI technique in connection with the study of PMSE. See La Hoz et al. (1989) for a technical information regarding the radar.

Here we outline some of the radar parameters that were set for the experiment. Data were collected for the altitude range of $77.85-95.85 \mathrm{~km}$ using a 64-baud complementary code with a $300 \mathrm{~m}$ range resolution. Pulses were transmitted with an inter-pulse period of $2.487 \mathrm{~ms}$, and 12 samples were coherently averaged to produce a single data point (in-phase and quadrature). A total of 64 data points were stored before writing the information to a magnetic tape. This resulted in a dwell time of $1.88 \mathrm{~s}$, but the data dump interval was $2 \mathrm{~s}$. The 64 pulses were evenly divided over the two frequencies, which corresponds to 32 real-time data points per frequency per dwell. A brief overview of the relevant settings for the experiment is given in Table 1 .

Table 1. Radar settings during the experiment

\begin{tabular}{ll}
\hline Parameter & Setting \\
\hline Frequencies & 224.0 and $224.6 \mathrm{MHz}$ \\
Sub-pulse length & $2 \mu \mathrm{s}$ \\
Range resolution & $300 \mathrm{~m}$ \\
Code & $64-$ bit complementary code \\
Code sequence & $\mathrm{A} \overline{\mathrm{B}} \mathrm{A} \mathrm{B}$ \\
Inter-pulse period & $2487 \mu \mathrm{s}$ \\
Coherent integrations & 12 \\
Minimum lag time & $29.85 \mathrm{~ms}$ \\
Time-series points & 64 \\
Dwell time & $1.88 \mathrm{~s}$ \\
Duty cycle & $5.15 \%$ \\
Range gates & 61 \\
\hline
\end{tabular}


The radar was operated in the mode described for a total period of $55 \mathrm{~h}$ from 25 July to 7 August, 1997 with the majority of the observations occurring between 2200 and 0200 UT. This time interval has been selected for historical reasons. That is, during previous PMSE/NLC campaigns involving the EISCAT VHF radar, observations were scheduled during these times for the sake of visual NLC observations and to improve the data quality of lidar observations. We continue to make observations during these intervals in order to test for yearly trends in the occurrence of PMSE. The FDI observations were conducted on 14 different occasions during the two week interval. Indications of PMSE, albeit weak at times, could be seen in the data for all but one of these occasions. We have chosen for closer examination, two cases in which the PMSE was strongest over the longest period of time. During these two events, only half of the antenna could be used due to technical problems with the transmitting klystrons. This affects the antenna beam width as discussed in the next section.

\section{Application of FDI to PMSE data}

Frequency domain interferometry is a radar technique developed to study structures in the atmosphere having a vertical extent smaller than that of the sampling volume. As we discuss later, this is accomplished by operating the radar at two or more closely spaced frequencies. The FDI technique has already been treated in several publications, for example (Franke (1990), Kudeki and Stitt (1990) and Franke et al. (1992). Therefore, we only briefly summarize the technique here. FDI makes use of the amplitude and phase of the normalized cross-correlation or cross-spectrum formed by frequency pairs. For the sake of illustration, assume that a radar signal is scattered in such a way that the bulk of the echo power originates from a single layer located within the radar sampling volume. Further assume that the the range resolution of the radar is $300 \mathrm{~m}$. This situation is depicted graphically in the left panel of Fig. 1, where the width of the layer is $30 \mathrm{~m}$. If two radar waves of different wavelength are transmitted (middle panel of Fig. 1), then the amplitude and phase of the received signal, which was scattered from the layer, can be used to estimate the width and position of the layer, respectively. In the example shown in Fig. 1, the phase difference between the received signals of different wavelengths would be about $300^{\circ}$ (right panel). Note that the same would be true if the layer were replaced by a steep, horizontally extended vertical gradient in the refractive index. The ability to accurately resolve the width and position of the layer will depend on the signal-to-noise ratio.

For our study of PMSE, we have adopted an FDI approach similar to that presented in Franke (1990) and as described in Chilson and Schmidt (1996). That is, we have completed our FDI analysis in the time domain using correlation functions. The discrete un-normalized complex cross-correlation function is calculated using

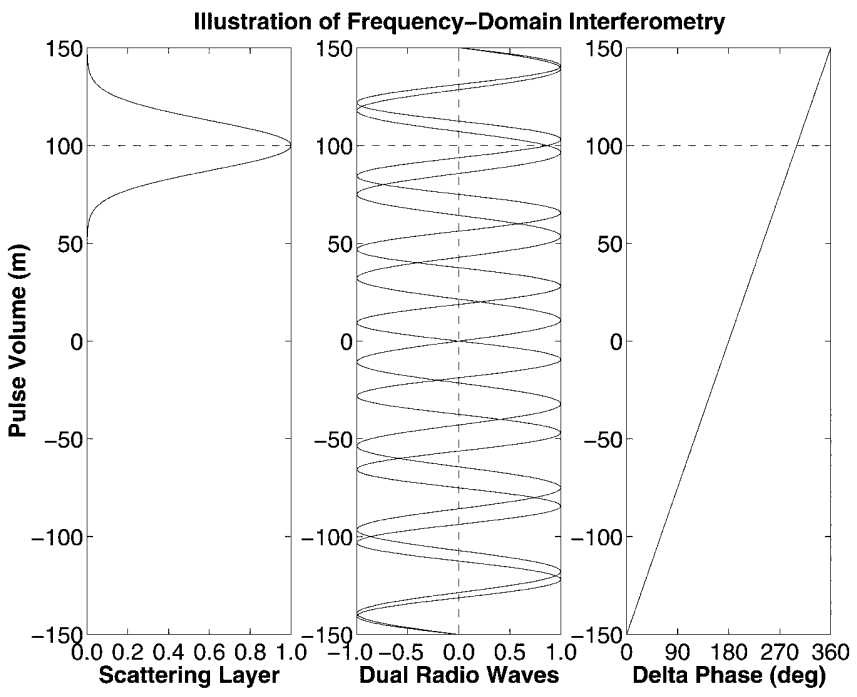

Fig. 1. Illustration of the basic principles for FDI. One possible example of the phase and amplitudes for two different carrier frequencies are shown in the middle panel. See the text for further details

$C_{12}(n)=\sum_{k=1}^{N} V_{1, k} V_{2, k+n}^{*}$

where $V_{1, k}$ and $V_{2, k}$ refer to complex time series data corresponding to frequencies $f_{1}$ and $f_{2}$, respectively. The index $k$ refers to individual samples within the time series and there are $N$ samples. The value $n$ denotes the number of lags. From Eq. (1) we calculate the complex correlation coefficient from the expression

$S_{12}=\frac{C_{12}(n=0)}{\sqrt{C_{11}(n=0) C_{22}(n=0)}}$.

According to Kudeki and Stitt (1990), $S_{12}$ is related to properties of a singular atmospheric scattering layer through the expression

$S_{12}=\mathrm{e}^{-i 2 \Delta k z_{l}} \mathrm{e}^{-\Delta k^{2} \sigma_{l}^{2}}$,

where $z_{l}$, is the relative height of the layer, $\sigma_{l}^{2}$ is the second central moment of the layer's thickness, and $\Delta k$ is the difference in wave number for the two radar frequencies. In deriving Eq. (3), the effects of having a finite beam width and resolution volume have not been taken into account. Furthermore, it is assumed that the height interval over which the phase differences between the two radar carrier frequencies span $2 \pi$ matches the range resolution being used. A more general expression is given as

$S_{12}=\mathrm{e}^{-i 2 \Delta k\left(z_{0}+2 z_{l} \sigma_{r}^{\prime 2} / \sigma_{l}^{2}\right)} \mathrm{e}^{-\Delta k^{2} \sigma_{r}^{\prime 2}}$,

where

$\sigma_{r}^{\prime}=\frac{\sigma_{l}^{2} \sigma_{r}^{2}}{\sigma_{l}^{2}+2 \sigma_{r}^{2}}$

(Franke, 1990). In these expressions, $z_{0}$ and $\sigma_{r}^{2}$ are the first and second moments, respectively, of the the vertical component of weighting function for the radar 
sampling volume. Likewise, $\sigma_{l}^{2}$ is the second central moment of the transverse component of the weighting function. See Doviak and Zrnić (1984) and Franke (1990) for further details. Equations (3) and (4) are similar for a thin scattering layers; however, width estimates of thicker layers are underestimated by Eq. (3). In Fig. 2 we show the dependence of the estimated layer width on coherence for the two models.

From Eq. (4), the first and second moments of the assumed Gaussian-shaped layer are calculated using

$z_{l}=\frac{-\left\langle S_{12}\left(2 \sigma_{r}^{2}+\sigma_{l}^{2}\right)\right.}{4 \Delta k \sigma_{r}^{2}}$

and

$\frac{1}{\sigma_{l}^{2}}=\frac{\Delta k^{2}}{-\ln \left[\left|S_{12}\right| \sqrt{\left(1+N_{1} / S_{1}\right)\left(1+N_{2} / S_{2}\right)}\right]}-\frac{1}{2 \sigma_{r}^{2}}$,

respectively, where $\angle S_{12}$ is the phase angle for $S_{12}$. As discussed by Kudeki and Stitt (1990) the estimate of the coherence given by Eq. (2) will be contaminated by noise. This effect is mitigated in Eq. (7) by weighing $\left|S_{12}\right|$ with $\sqrt{\left(1+N_{1} / S_{1}\right)\left(1+N_{2} / S_{2}\right)}$, where $S$ and $N$ denote the signal and noise estimates, respectively, recorded at the two different frequencies.

Of course, coherence values that we obtain from Eq. (2) are only an estimate of the true coherence, and the estimate will only be meaningful if it can be shown to be statistically significant. If we take as a null hypothesis that the true coherence is zero, then the $95 \%$ significance level for a coherence estimate being non-zero is given by

$S_{0.95}=\left[1-(0.05)^{1 /(\gamma-1)}\right]^{1 / 2}$,

where $\gamma$ is the number of degrees of freedom (Franke et al., 1992). Since the correlation time of the atmosphere may be larger than the interval between data samples, the number of degrees of freedom should be calculated using

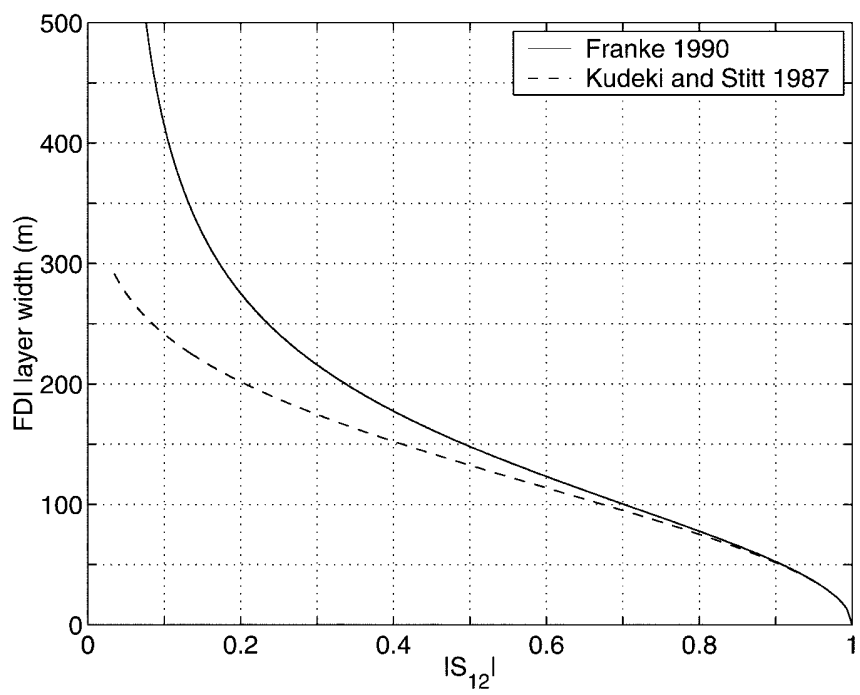

Fig. 2. Dependence of the FDI layer width on $\left|S_{12}\right|$ based on two different models $\gamma=1+\left(\frac{2 \ln 2}{\pi}\right)^{1 / 2} \frac{T}{\tau_{1 / 2}}$.

Here $T$ is the time span of the data used in calculating $S_{12}$ and $\tau_{1 / 2}$, is related to the correlation time of the atmosphere (Chilson and Schmidt, 1996). Note that these equations correct a typesetting error in Eq. (6) of Chilson and Schmidt (1996).

We should point out that there are inherent limitations of any two-frequency FDI analysis. Most importantly, the original derivation of the FDI analysis assumed one Gaussian-shaped weighting function for the scattering layer per sampling volume. If several dominating layers are present, then the position and width estimates from the two-frequency FDI analysis provide only the first and second moments of the vertical reflectivity structure within the pulse volume. Methods for overcoming this limitation are being investigated (Palmer et al., 1999) and could be applied to the study of PMSE in future work. However, as discussed by Chilson et al. (1997b), even if several scattering layers are present within a radar sampling volume then an FDI analysis can still provide an indication as to when and where layered structures occur.

An important consideration when applying FDI to PMSE observations is that of phase fronts. If all phase fronts of a propagating radar pulse at time $t$ are assumed to be at the same height, then a flat scattering layer (plane) will appear "warped" in the reference frame of the sampling volume (see Fig. 3). This leads to phase mixing across the radar sampling volume and a reduction of the correlation coefficient even if a single thin scattering layer is present. The degree of phase mixing is related to the radar beam width. Whereas this effect poses no serious problems to measurements made by MST radars in the troposphere and lower stratosphere, it can present a serious limitation for measurements made in the mesosphere. A discussion of the applicability of FDI measurements under various conditions can be found in Franke (1990).

The EISCAT VHF radar's antenna is a partial parabolic cylinder extending over $120 \mathrm{~m}$ in the zonal

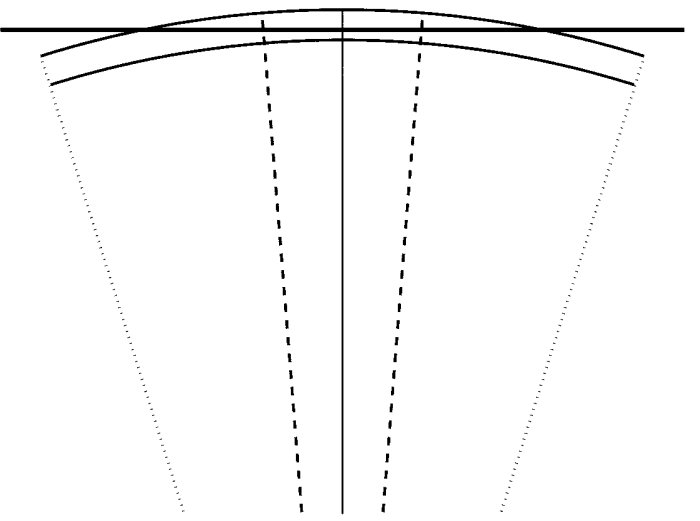

Fig. 3. Illustration of the apparent layer distortion resulting in beamwidth effects 
direction and $40 \mathrm{~m}$ in the meridional direction. During our experiment, power from one klystron (1 MW) of two was channelled through half of the antenna $(60 \mathrm{~m} \times 40 \mathrm{~m})$ for transmission while the entire antenna was used for reception. The resulting half power beam width was $1.7^{\circ}$. The antenna was pointing vertically during the observations. As illustrated in Fig. 3, if the radar beamwidth is large (dotted lines), then the amount of warping can be correspondingly large. Using the beamwidth of the EISCAT VHF radar (dashed lines), we find that at a height of $88 \mathrm{~km}$ a layer will experience a maximum apparent warping of only about $10 \mathrm{~m}$. This is $3 \%$ of the $300-\mathrm{m}$ range resolution used in our experiment. For comparison we calculate the amount of apparent warping that would be experienced for a radar having a half-power beam width of $5^{\circ}$. In this case the value is $84 \mathrm{~m}$, or $28 \%$ of the range resolution.

\section{Data analysis and overview}

We now begin a discussion of how the time-series data have been processed for the current analysis. The data records, which contain interlaced measurements made at the two frequencies, were divided over 64 complex timeseries points. That is, there are 32 points per frequency. Note that the time separation between data points of the same frequency is $59.70 \mathrm{~ms}$, which corresponds to a Nyquist frequency of $8.38 \mathrm{~Hz}$, or a Nyquist velocity of $5.61 \mathrm{~m} \mathrm{~s}^{-1}$. After separating the two frequencies, 10 contiguous records were appended to create 20-s long data streams containing 320 complex data points each. DC offsets and outliers were then removed from the in- phase and quadrature components of the time series, respectively. This was done separately for each frequency. Outliers were identified as those points having a magnitude larger than 5 standard deviations of a particular 320-point time series. The resulting preprocessed in-phase and quadrature data were used for the FDI analysis.

In addition to being used for FDI analysis, each of the 20-s long data streams were processed using a conventional moments method calculation (Woodman, 1985). That is, estimates of the signal strength together with line-of-sight velocity and spectral width were calculated from each data stream and for each frequency. Spectra were calculated using the 32-point sub sequences within the longer 20 -s long data stream. So, each of the estimates represents 10 incoherent integrations. We will use the symbols $\omega$ and $\sigma^{2}$ to represent the first and the second central moments, respectively, of the Doppler velocity.

We have chosen to focus on those data collected during the nights of August 02/03 and August 03/04, 1997. The PMSE data recorded on these two nights are well suited to our analysis in that they were both strong and persistent. Furthermore, these particular radar echoes serve well to illustrate interesting wave activity in the atmosphere. The results of the moments calculation for the data are shown in the overview plots in Figs. 4 and 5 . We will refer to these and similar plots as range-timepseudocolor (RTP) plots. Note that in the RTP plots of $\omega$ and $\sigma$, only those values are shown for which the corresponding SNR was larger that $8 \mathrm{~dB}$. Similar to Hocking and Röttger (1997), this threshold was chosen to assure that the data were not biased by noise.
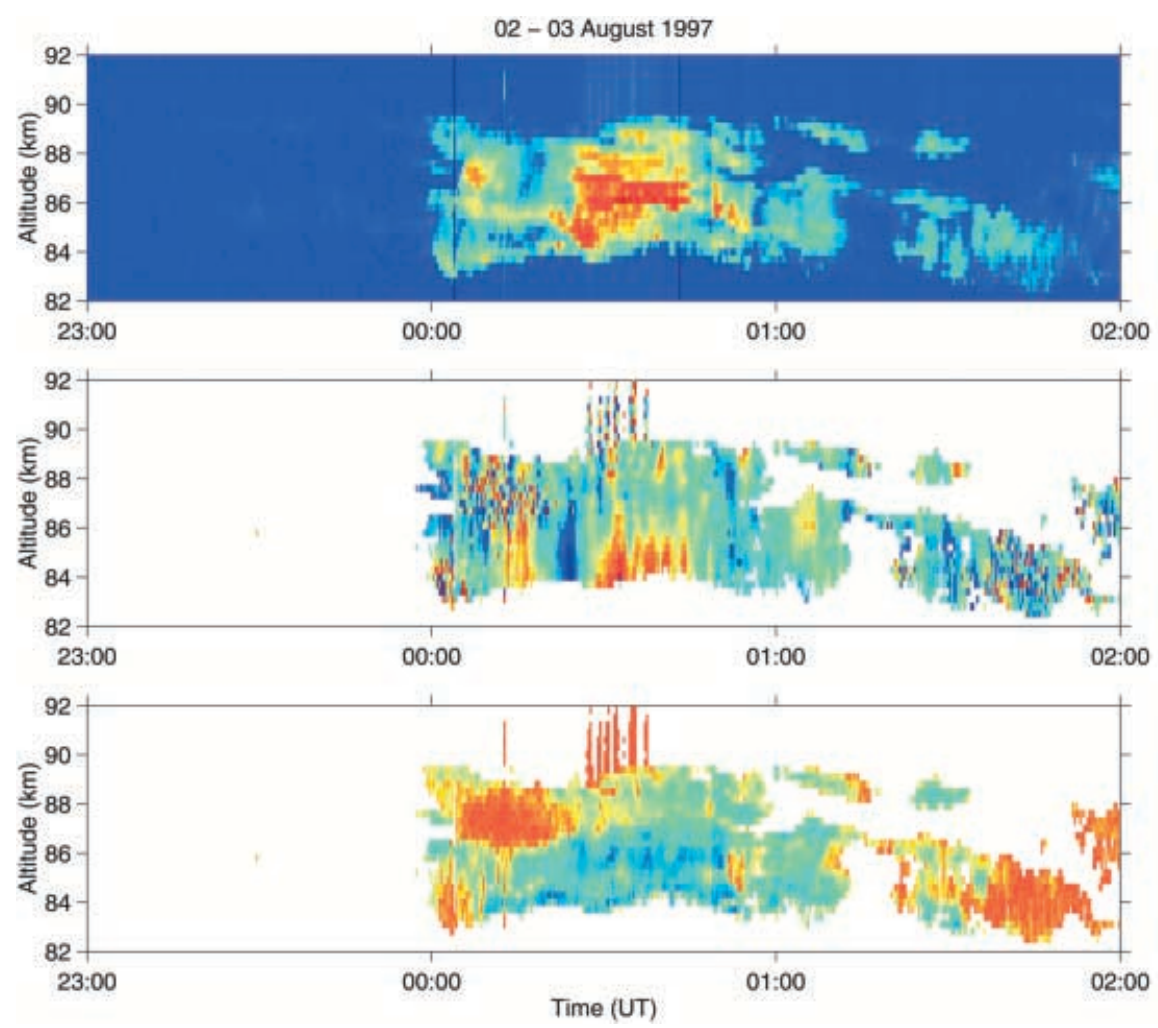
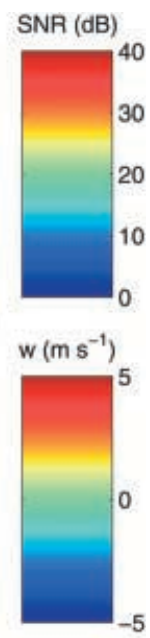

$\sigma\left(\mathrm{m} \mathrm{s}^{-1}\right)$

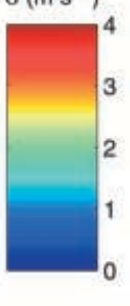

Fig. 4. Range-time-intensity plots of SNR, radial Doppler velocity, and the Doppler spectral width 


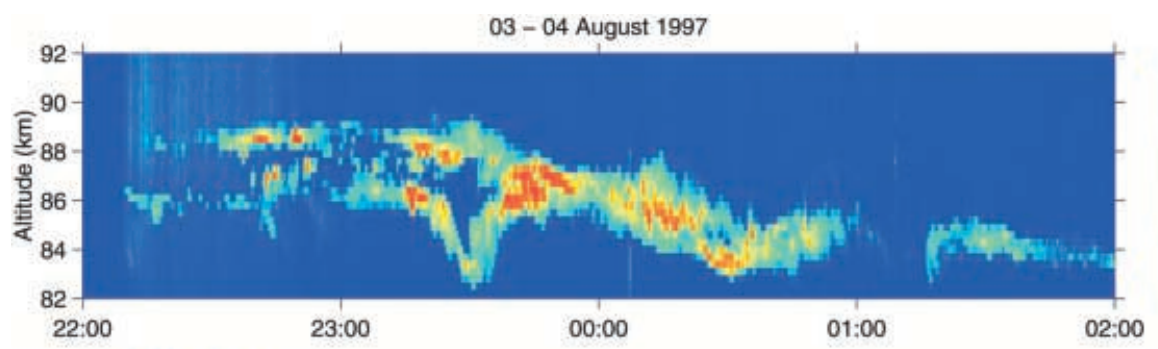

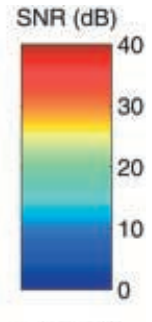

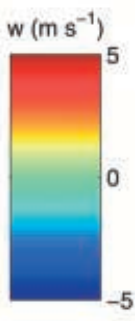

$\sigma\left(\mathrm{m} \mathrm{s}^{-1}\right)$

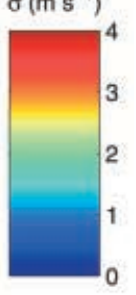

Fig. 5. Same as Fig. 4 but for 03-04 August, 1997

It is possible to identify several interesting features in the RTP plots of Figs. 4 and 5 even before beginning the FDI analysis. The general appearances of the two PMSE events are quite different; however, both show evidence of strong background dynamics in the atmosphere. During the first PMSE event (Fig. 4), large oscillations in the vertical velocity as a function of time can be seen, especially between 0:00 and 01:00 UT. Evidence of these oscillations in the vertical wind field is not so apparent in the RTP plot of SNR. For the second PMSE event (Fig. 5), a signature of atmospheric dynamics is more evident in the RTP plot of SNR. However, in contrast to regular modulations of the PMSE layer height, for example, as shown by Rüster et al. (1996) and Rüster (1997), these data indicate what may be a singular, large amplitude wave disturbance between 23:00 and 00:00 UT.

Another feature common to both PMSE events is the presence of localized cells of enhanced spectral width $(\sigma)$. Examples of these can be seen in Figs. 4 and 5 between approximately 00:00 and 00:30 UT on both August 3 and August 4. These regions, which are most probably associated with strong atmospheric turbulence, may have been generated by a Kelvin-Helmholtz (KH) mechanism. We note that other factors besides turbulence can contribute to Doppler spectral widths. These include beam broadening, shear broadening, and gravity-wave broadening (see Nastrom and Eaton, 1997, and references therein). Of these, beam broadening is typically the dominating element. Due to the narrow beamwidth of the EISCAT VHF radar, even horizontal winds of $80 \mathrm{~m} \mathrm{~s}^{-1}$ only contribute to the spectral widths just over $1 \mathrm{~m} \mathrm{~s}^{-1}$ (Hocking and Röttger, 1997).
Czechowsky and Rüster (1997) reported similar cloud-like structures of enhanced turbulence in PMSE data, which they claim to be associated with KH dynamics. They characterize these cells as: appearing predominantly in the upper portion of the PMSE; not being correlated with echo power; and having lifetimes of 20 to $30 \mathrm{~min}$. Their description also applies for the cells of enhanced spectral width shown in Figs. 4 and 5. It is not clear from our observations whether these cells are causally related to a $\mathrm{KH}$ mechanism; however, in Sect. 5 we present additional evidence that supports the supposition that $\mathrm{KH}$ dynamics are active during these times.

So far we have only been discussing the spectral moments associated with the strongly enhanced backscatter of the PMSE. However, the background levels of SNR, those not associated with the PMSE, also reveal a time variability. In particular, an increase in the background level of SNR can be seen near 00:30 UT in Fig. 4 and between 22:00 and 23:00 UT on 3 August in Fig. 5. This is believed to result from particle precipitation, which enhances the electron density. Precipitating particles could also be linked with the production of PMSE, which require free electrons in the D-region. One might expect sufficient numbers of electrons to be present near the summer polar mesopause for the generation of PMSE without particle precipitation. However, the levels may be below the limits of saturation in the late summer months around local midnight (Cho and Röttger, 1997). If this is the case for the times of data shown in Figs. 4 and 5, then precipitating particles could have an effect on the PMSE events. We do note that the signal strength of the first PMSE increases around 00:30 
on August 3. Also, the second PMSE event is activated shortly after the time of the second presumed case of precipitating particles.

We now consider data from a relative ionospheric opacity meter (riometer), which can be used to detect the presence of particle precipitation. The riometer is a passive radio receiver, typically operating in the frequency range of 20 to $50 \mathrm{MHz}$, that monitors the intensity of cosmic radio noise. During periods of increased ionization in the D- and E-regions, the cosmic radio noise is absorbed, resulting in a reduction of signal being received by the riometer. Absorption at high latitudes is generally caused by the precipitation of energetic electrons from the magnetosphere, which increases the ionospheric electron density between about 70 and $120 \mathrm{~km}$ altitude. We have taken data from IRIS (imaging riometer for ionospheric studies) located at Kilpisjärvi $\left(69.05^{\circ} \mathrm{N}, 20.79^{\circ} \mathrm{E}\right)$. The IRIS site is located $85 \mathrm{~km}$ east-southeast of the EISCAT radar. Figure 6 shows the total absorption curve over all beams for the entirety of 3 August, 1997. See http://www.dcs.lancs. ac.uk/iono/ for additional information about the IRIS facility. The intervals corresponding to the enhanced background levels of SNR have been flagged by vertical lines. Both of these periods correspond to periods of enhanced absorption. Therefore, it is conceivable that in these cases the presence of precipitating particles had an influence on the backscattering cross section of the PMSE.

Next we present the results of processing the data shown in Figs. 4 and 5 using FDI. For the analysis, the pre-processed 20-s data streams were taken as the values of $V$ in Eq. (1) when calculating $S_{12}$. These data streams

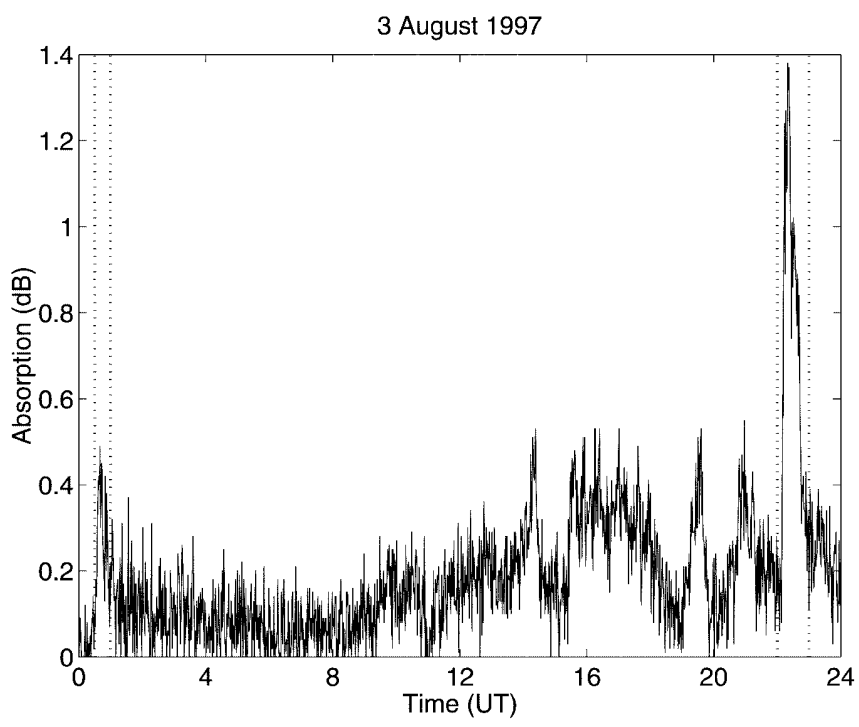

Fig. 6. Absorption data from IRIS (imaging riometer for ionospheric studies) located at Kilpisjärvi $\left(69.05^{\circ} \mathrm{N}, 20.79^{\circ} \mathrm{E}\right)$. The vertical lines mark particular times for which particle precipitation is anticipated

provided good time resolution while still assuring adequate confidence levels for the correlation coefficients. From $S_{12}$, the values of $z_{l}$ and $\sigma_{l}$ were calculated using Eqs. (6) and (7). These quantities are presented in Figs. 7 and 8. Only those cases for which the coherence was larger than the $95 \%$ significance level are shown. Note that the range of layer width in the RTP plots extends from 100 to $300 \mathrm{~m}$. There were few cases in which the widths were smaller than $100 \mathrm{~m}$.
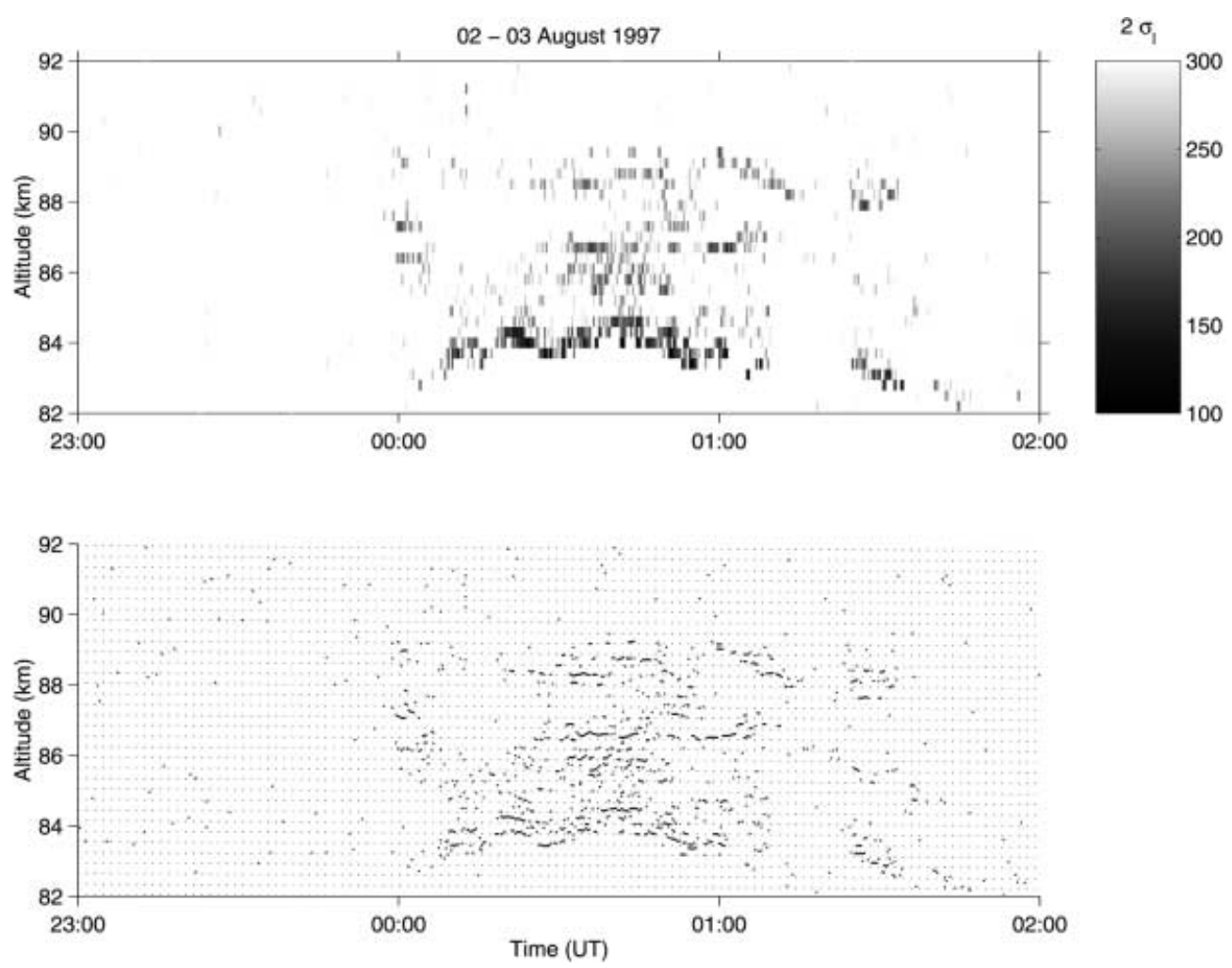

Fig. 7. Range-time-intensity plots of FDI layer width as given by $2 \sigma_{l}$ (upper panel). The FDI estimates of the layer positions (lower panel). Also shown are reference points (dotted lines) delineating the upper and lower boundaries of the conventional radar range resolution 
In the remainder of this section, some of the largescale features of the FDI data are discussed. A more detailed analysis is given in Sect. 5. We begin with those data presented in Fig. 7. It is notable that the thicknesses of the scattering layers identified by FDI are smallest at the boundaries of the PMSE layers. In fact, the smallest widths are located near the bottom of main PMSE layer at a height of about $84 \mathrm{~km}$; however, coherent scattering layers are also present at the top of the PMSE. An internal structuring within the PMSE is evident from the lower panel of Fig. 7. One can easily follow the undulations in the estimates of $z_{l}$, which presumably are tracing the vertical displacements of the layers in time.

At this point we should note that the the frequency separation of $600 \mathrm{kHz}$ used in this experiment inherently introduces a potential range aliasing in the FDI estimates of layer position. As demonstrated in the right-hand panel of Fig. 1, it is possible to choose the frequency separation of the two carrier frequencies such that phase angle of the $S_{12}$ spans a range of $2 \pi$ over a height interval that matches the radar range resolution. For a frequency spacing of $\Delta f=600 \mathrm{kHz}$, the phase angle sweeps through the range of $2 \pi$ every $250 \mathrm{~m}$. Therefore a scattering layer located near the lower (upper) boundary of the sampling volume will be aliased to the upper (lower) portion of the same volume.

In Fig. 8, it is more difficult to identify a correlation in the location of the PMSE layer and regions characteristic of having scattering structures with narrow widths. This could be related to the more dynamic nature of the PMSE layer itself. There are, however, regions demonstrating the same correlation as in the data shown in Fig. 7. We can also observe that there is no clear relationship between the signal strength and the layer width. These points become more evident in Sect. 5.

\section{Vertical structure within the PMSE}

Although RTP plots are useful when examining the morphology of the PMSE structures, height profiles are required to study small-scale features. In Figs. 9 and 10, consecutive profiles of SNR, coherence, and confidence level are presented. Each of the profiles has been calculated from 20 -s long data segments and the profiles of SNR have been scaled for the sake of plotting. To retrieve the original SNR values, in decibels, the scaled values shown must be multiplied by 40 . The profiles in the left-most panel of Fig. 9 were calculated from data near the onset of the PMSE and those in the right-most panel correspond to the time when the echoes began to weaken. The time separation between consecutive sets of profiles in the time series is approximately $17 \mathrm{~min}$. The start and stop times in Fig. 10 were simply chosen so as to provide coverage over most of the PMSE without having too large of a time interval between consecutive sets of profiles. For Fig. 10 the time separation is approximately $20 \mathrm{~min}$.

It was already mentioned in Sect. 4 that coherent scattering layers are observed at heights corresponding to the lower boundary of the PMSE. This feature is particularly evident in the profiles shown in Fig. 9. A
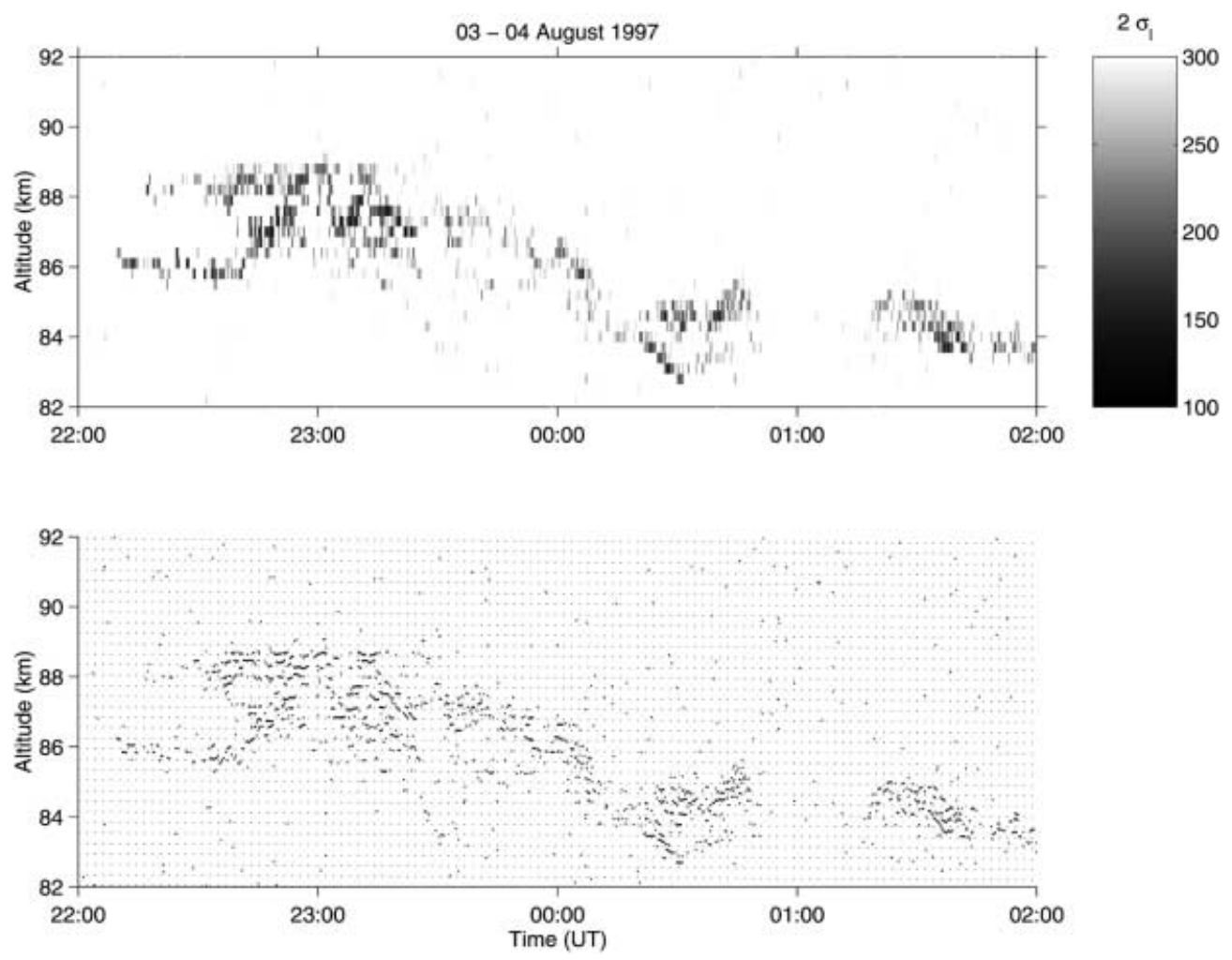

Fig. 8. Same as Fig. 7 but for 03-04 August, 1997 

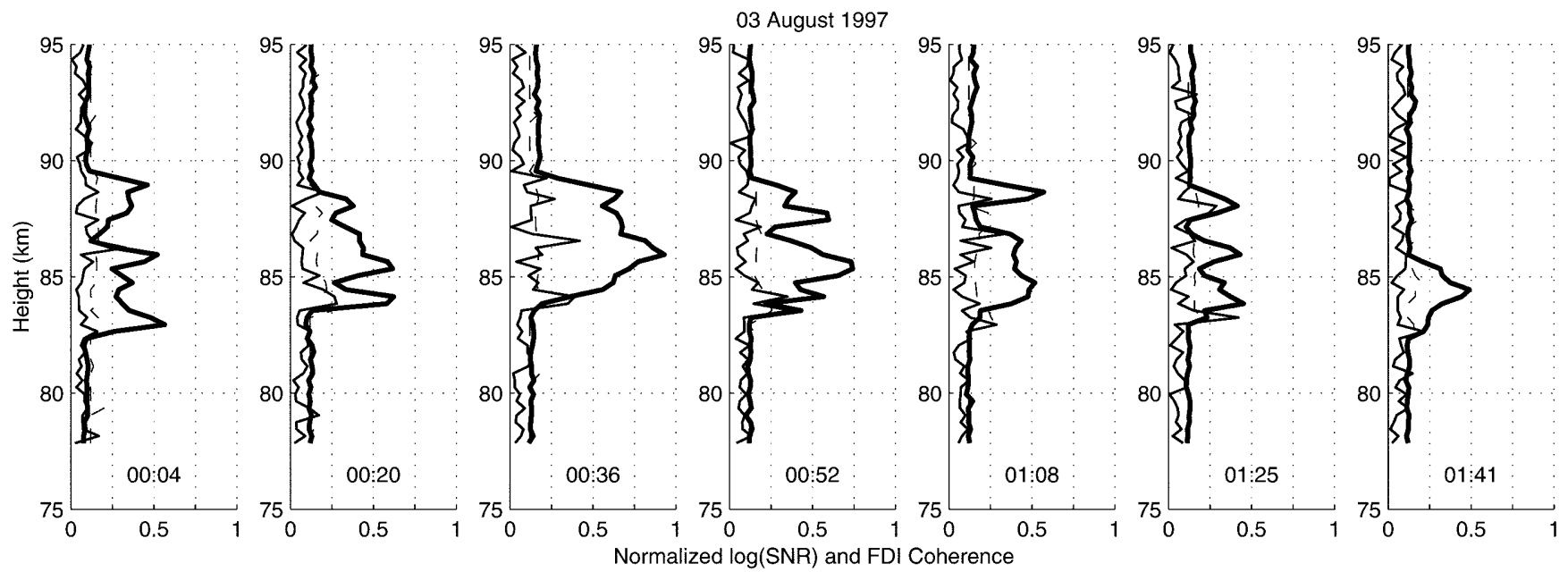

Fig. 9. Profiles of the normalized SNR (heavy solid line), coherence $\left|S_{12}\right|$ (light solid line) and the $95 \%$ significance level of $\left|S_{12}\right|$ (dashed line). The SNR values expressed in decibels have normalized by

dividing by 40 . The time interval between consecutive profiles have been chosen to be close to constant. For reference, the times for the individual profiles expressed in UT are shown in figure panels
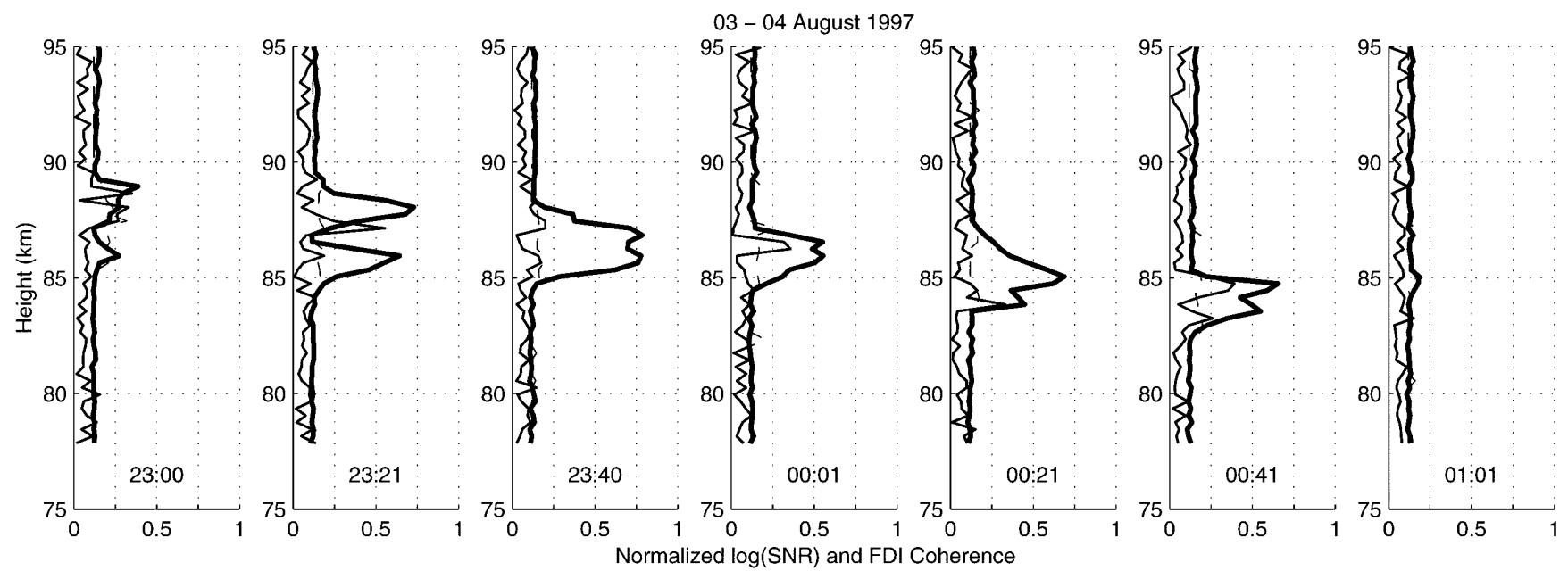

Fig. 10. Same as Fig. 9

comparison of several such profiles (not shown here) reveals that a region of high coherence delineates the lower boundary of the PMSE layer extremely well. Indeed, it is not unusual to observe that the coherence values are peaked around the heights corresponding to both the lower- and upper-most boundaries of the PMSE layer. As in Fig. 9, there are also several cases in Fig. 10 for which the coherence peaks on the upper or lower boundaries of the PMSE layers or sub-layers. Considering the frequency of occurrence of the relative positioning of the peaks in coherence and SNR, we are led to consider a mechanism responsible for it.

One possible mechanism may be related to the presence of $\mathrm{KH}$ instabilities. High temporal- and spatial-resolution simulations of such instabilities have been recently reported by Werne and Fritts (1999). They have calculated height profiles of the first four statistical moments of the temperature and the streamwise background flow through a KH instability layer. The vertical gradient of the mean temperature is positive in the upper and lower boundaries of the instability layer and close to zero within the layer. Extended layers of anisotropic turbulence could form at these boundaries (Woodman and Chu, 1989). Hocking and Röttger (1997) discuss the relevance of this and other mechanisms for the layering and aspect sensitivity found within PMSE.

Results of KH instability simulations have been directly applied to the study of PMSE by Hill et al. (1999). They compare height profiles of the dimensionalized potential temperature from $\mathrm{KH}$ simulations with rocket data of electron number densities from the STATE experiment, see their Fig. 4. Here it is shown that steps in the height profile of the electron number density correspond with the edges of the instability layer, which was about $1 \mathrm{~km}$ thick. Therefore, the enhancements in coherence that we are observing may result from horizontal structuring of the plasma as a 
result of $\mathrm{KH}$ instabilities. We note that Hocking and Röttger (1997) have shown that partial reflection may occur from large-scale ledges in the electron number densities. However, we are not suggesting that the steps in electron concentrations are responsible for the echoes that we are seeing. Furthermore, Hocking and Röttger (1997) did not exclude the possibility of specular reflections associated with PMSE resulting from sharp edges on turbulent layers or highly anisotropic scatterers at the edges of turbulent layers.

$\mathrm{KH}$ instabilities form in regions of large vertical shear in the horizontal wind. We only have measurements for the vertical component of the wind from our EISCAT radar data. Therefore, we have acquired wind measurements from the ALOMAR SOUSY VHF radar (Singer et al., 1995), which is located about $125 \mathrm{~km}$ west of the EISCAT radar. These data were also collected during periods of PMSE. In Fig. 11 we show profiles of the zonal and meridional wind components. The wind data have been averaged in time and height over intervals of $1 \mathrm{~h}$ and $900 \mathrm{~m}$, respectively. It is clear that the horizontal wind is strongly sheared.

One means of parametrizing the dynamic stability of flows in the atmosphere is to calculate the gradient Richardson number, $R i$. On the grounds of theoretical considerations, a necessary but not sufficient condition for the production of $\mathrm{KH}$ instabilities is that $R i \leq 0.25$ (Miles and Howard, 1964). The gradient Richardson number can be calculated through the expression

$R i=\left[\frac{g}{\bar{\theta}} \frac{\Delta \theta}{\Delta z}\right] /\left[\left(\frac{\Delta u}{\Delta z}\right)^{2}+\left(\frac{\Delta v}{\Delta z}\right)^{2}\right]$,

where $g$ is the gravitational acceleration, $\theta$ is the potential temperature, $z$ is the altitude, and $u$ and $v$ are the zonal and meridional components of the wind, respectively. We have calculated $R i$ using the wind data
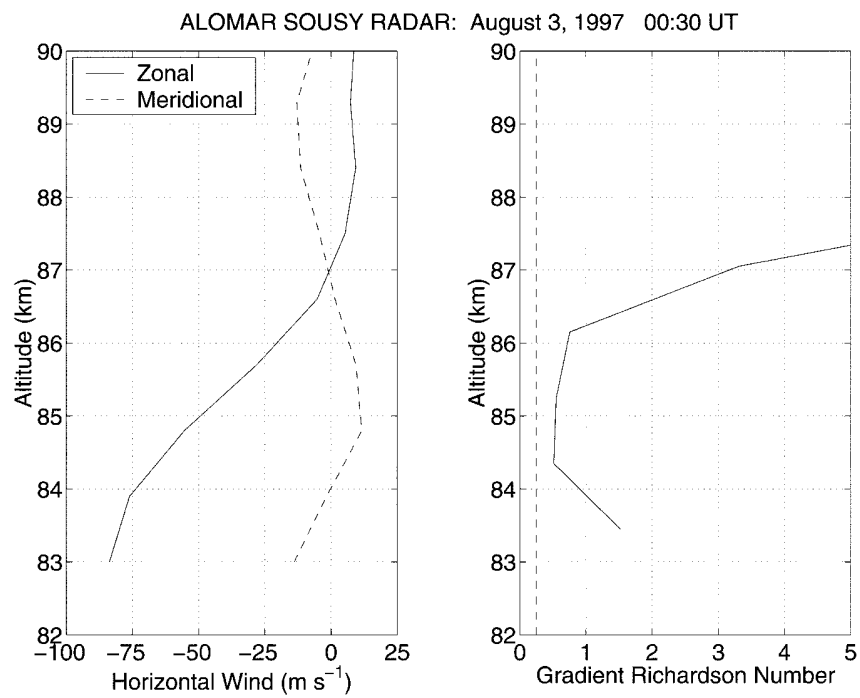

Fig. 11. Left panel: hourly averaged horizontal wind measurements from the ALOMAR SOUSY VHF radar. Right panel: estimate of the Richardson number based on the horizontal wind data and profiles of temperature and density given in Lübken (1999) from the ALOMAR SOUSY radar and calculations of $\theta$ based on the measurements of temperature and density given in Lübken (1999). The profile of $R i$ is shown in Fig. 11. The minimum in the profile corresponds to the altitude of the lower PMSE sublayer discussed earlier. We should be cautious when interpreting the exact values of $R i$, since they were calculated from averaged wind data from a different radar site and the potential temperatures are based on profiles of temperature and density taken from Lübken (1999). Nevertheless, the profile does support the assumption that $\mathrm{KH}$ instabilities are responsible for some of the features that we have seen in our data.

Lastly, we note that the line profiles of coherence in Fig. 9, as well as the RTP plot of layer width in Fig. 7 also provide evidence of sub-structuring within the PMSE layer. In Fig. 9 we find that the coherence obtains a maximum value at a height of approximately $86 \mathrm{~km}$ at 00:36 UT. This is in the center of the PMSE layer and possibly marks the location of a bifurcation. That is, the local maximum in the coherence could correspond to the upper (lower) boundary of a (upper) lower sub-layer. An increase in the value of coherence marks a decrease in the FDI estimate of layer width, and we note that a persistent layer having a width of less than $300 \mathrm{~m}$ can be found in Fig. 7 near a height of $86 \mathrm{~km}$.

An additional indication that the large coherence values at $86 \mathrm{~km}$ mark the boundary between two sublayers is found in the RTP plots of the vertical velocity and spectral width in Fig. 4. Between 00:00 and 01:00 UT, these quantities are distinctly different in the upper and lower portions of the PMSE layer. Above the altitude of $86 \mathrm{~km}$, the vertical velocities follow a wave pattern that exhibits much more temporal variability than below this level. Additionally, the spectral widths are larger in the upper portion of the layer. This is consistent with earlier studies, which have shown the lower portions of PMSE layers to be aspect sensitive and to be associated with regions having very little neutral turbulence (Cho et al., 1993; Lübken et al., 1993). It is possible that the prevailing dynamics in the lower portions of some PMSE layers are quite different than in the upper portions, e.g., if influenced by $\mathrm{KH}$ instabilities.

\section{Indications of steepened gravity waves}

We now bring various aspects of our data together in an attempt to learn more about the dynamics of the atmosphere during the two PMSE events. In Figs. 4 and 5 we have shown the RTP plots of the calculated spectral moments. Another method of presenting spectral moment data for the study of PMSE was introduced by Röttger et al. (1988). They used self-normalized spectrograms to study the dynamic variability of the Doppler spectra. Further applications of spectrograms to PMSE studies can also be found in Röttger and $\mathrm{Hoz}$ (1990), Röttger et al. (1990) and Franke et al. (1992). It has been made apparent in these studies, which were all 
conducted using data from the EISCAT VHF radar, that sharp discontinuities in the time histories of Doppler frequencies for PMSE can often be observed. For example, one can observe sudden shifts or "jumps" in the centroid of the spectrum of Doppler frequencies that are larger that the width of the spectrum itself. The time interval during which the jumps occur is $10 \mathrm{~s}$ or so.

An explanation for the jumps has been proposed by Röttger et al. (1990). They maintain that these discontinuities result when a thin scattering layer, that has been transported vertically by a steepened wave, is advected horizontally through the radar sampling volume. As atmospheric gravity waves propagate upwards, they experience an increase in amplitude. Once the amplitudes become large enough, they typically begin to break. However, the waves can become saturated, in which case they release just enough energy into turbulence to maintain a constant amplitude (Fritts et al., 1988). The waves can also exhibit a nonlinear transfer of energy from the fundamental wave frequency to higher frequency harmonics, in which case the wave amplitude continues to increase but the structure of the wave becomes steepened or tilted (Mobbs, 1985; Weinstock, 1986).

It has been noted by Röttger et al. (1990) that evidence of wave steepening can be found in photographic images of NLC. They made reference to the time sequence of the bottom topography of NLC layers shown in Fig. 9 of Witt (1962). A reproduction of the figure can also be found in Röttger et al. (1990) as Fig. 8. The NLC layer is claimed to have been modulated by a steepened wave. There have been studies which link observations of NLC with PMSE (Nussbaumer et al., 1996; von Zahn and Bremer, 1999; Stebel et al., 2000). We note that a structure very similar to the one shown in Witt (1962) and discussed by Röttger et al. (1990) can also be seen in the RTP plot of SNR in Fig. 5. That is, the lower portion of the PMSE layer at approximately 23:30 UT exhibits an abrupt adjustment in altitude of about $1.5 \mathrm{~km}$.

We have calculated spectrograms for three selected cases from our PMSE data. These are intended to show the relationships between such quantities as SNR, coherence, vertical velocity, spectral width, and the spectral jumps. In Figs. 12-14, we show the spectrograms for particular times and heights. Accompanying the spectrograms are the corresponding time histories of the SNR and coherence, $\left|S_{12}\right|$. We additionally show the time histories of FDI estimates of the layer positions, $z_{l}$, calculated for the cases in which the coherence is larger than the $95 \%$ confidence level. Another means of estimating the altitudes of the scattering layers within the PMSE is by integrating the measured Doppler velocities with time. That is, we can calculate

$z_{n}=z_{k}+\sum_{j=k+1}^{n} w_{j} \cdot \Delta t$

where $z_{n}$ is the altitude of the layer at time step $n, z_{k}$ is some assumed altitude of the layer at time step $k, w_{j}$ is the measured Doppler velocity for time step $k$, and $\Delta t$
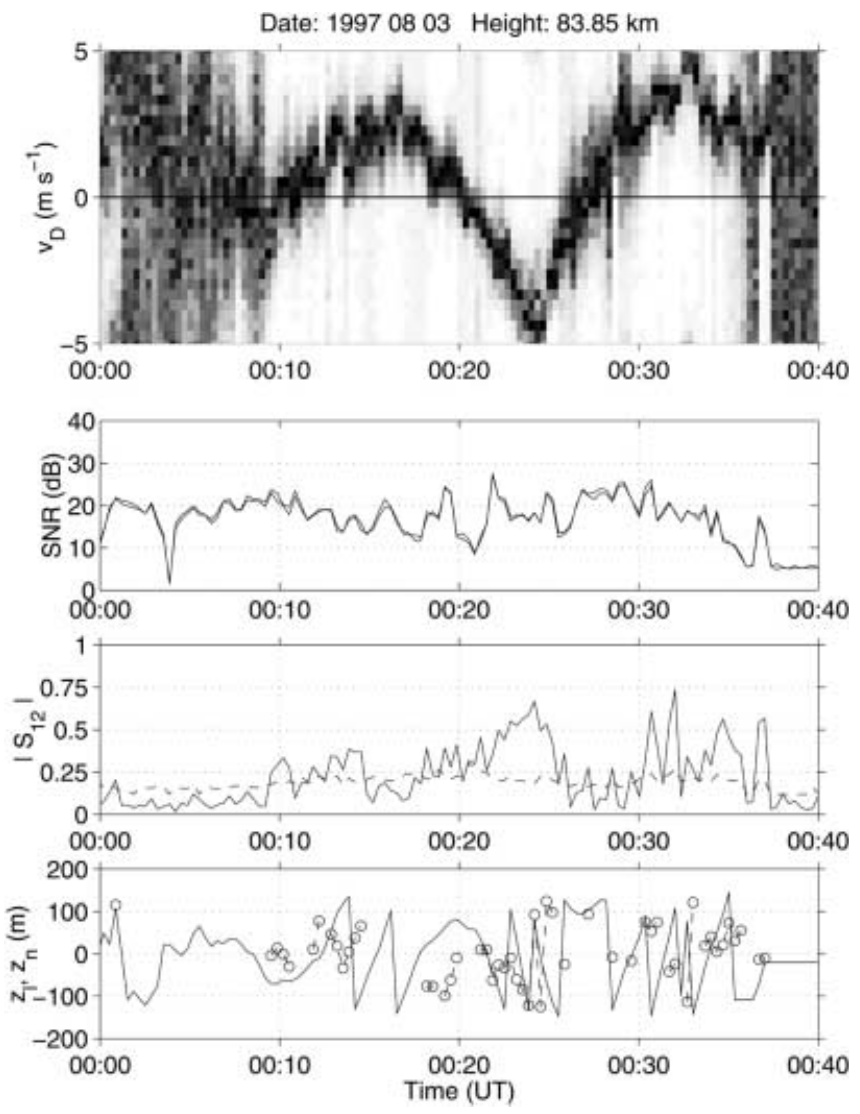

Fig. 12. The upper most panel shows the self-normalized spectrogram of Doppler velocities $\left(v_{D}\right)$. The second panel shows traces of SNR for both frequencies plotted separately. The third panel shows traces of the FDI coherence (solid line) and the 95\% significance level (dashed line). The fourth panel shows the trace of $z_{l}$ (circles) and $z_{n}$ (solid line)

is the time interval between consecutive estimates of $w_{j}$. Time histories of $z_{n}$ are shown in conjunction with $z_{l}$. Here we assume, as did Röttger et al. (1990), that vertical motions due to advection dominate over temporal variations of vertical movement. For each of the cases shown in Figs. 12 and 13, the value of $z_{k}$ has been set to zero. The values of $z_{n}$ are allowed to wrap about $\pm 150 \mathrm{~m}$ to keep them on the same scale as the values of $z_{l}$.

The first case to be considered in shown is Fig. 12 . The spectrogram does not exhibit a jump in the Doppler velocity, but we do find a cusp at about 00:24 UT. Although not shown here, this signature is evident in the spectrograms from the lower edge of the PMSE to a height of about $86 \mathrm{~km}$. Above this height, which was shown in Sect. 5 to represent a boundary between substructures, the cusp feature in the spectrogram begins to break up. A cusp in the spectrogram could result if a layer of scatterers, distorted in height, were advected horizontally through the radar beam. The cusp would correspond to a steeply sloped inflection point in the relative change of the layer's height with time. This, in turn, may result from a steepened wave.

Note that in the lower panel of Fig. 12 the values of $z_{n}$ vary in time much more rapidly than those of $z_{l}$. It is possible for the horizontal component of the wind to 

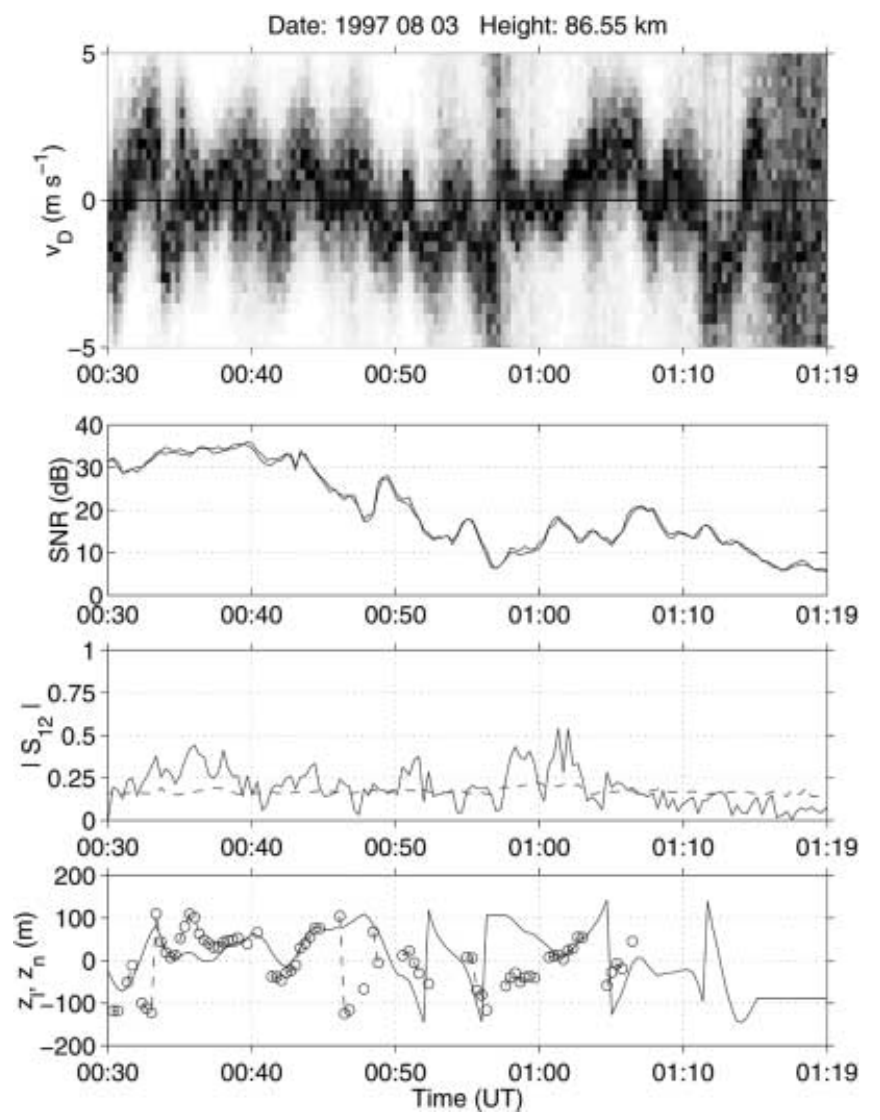

Fig. 13. Same as Fig. 12 but for a height of $86.55 \mathrm{~km}$ and for the time period 00:30-01:20 of 3 August, 1997

"leak" into the estimate of the vertical component of the wind. This can occur when aspect sensitive scattering layers are "tilted" with respect to a nominal horizontal orientation (Zecha, 1999). From Fig. 11 we find that the magnitude of the horizontal wind at this altitude was approximately $80 \mathrm{~m} \mathrm{~s}^{-1}$. An apparent deflection in the radar beam's zenith angle of $3^{\circ}$ would result in a bias in the estimate of $w$ of $4 \mathrm{~m} \mathrm{~s}^{-1}$. It is not clear, however, whether the disparity in the estimates of $z_{l}$ and $z_{n}$ is due to such contamination of the values of $w$ or whether the FDI estimates are biased towards zero by the rapidly ascending/descending layer position.

It is interesting to note that the coherence in Fig. 12 is peaked at the time when the Doppler velocity is most negative, that is, when the position of the layer is moving downwards most rapidly. A downward layer propagation is also reflected in the $z_{l}$ data. This may be coincidental; however, the data may be revealing a phase in the layer dynamics for which the atmosphere is locally stable. That is, the motions of the layer are such that the change in atmospheric density with time is positive and the local conditions are dynamically stable (Mobbs, 1985; Weinstock, 1987). In other words, the downward propagation of the layer supports well-defined, stably stratified structures that, in turn, lead to the high coherence values.

Next we present data for which jumps in the spectrogram are evident. These are found in Fig. 13.
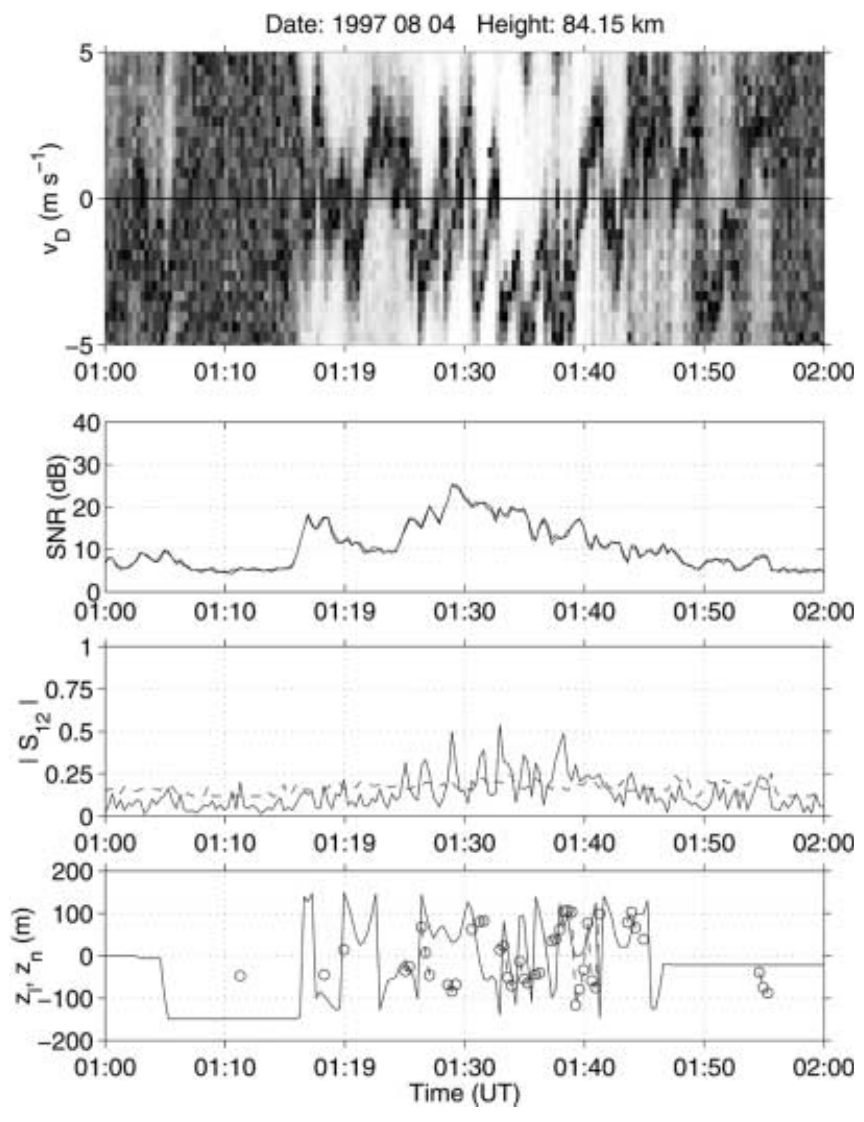

Fig. 14. Same as Fig. 12 but for a height of $84.15 \mathrm{~km}$ and for the time period 01:00-02:00 of 4 August, 1997

Two cases for which the spectral jumps are particularly evident occur at 00:33 and 00:58 UT; although, abrupt changes in the Doppler velocity can also be located for other times in the spectrogram. The Doppler velocities are mostly centred about zero. In that context, we draw attention to the lower panel of Fig. 13, where we find that the traces of $z_{l}$ and $z_{n}$, for this example, show very good agreement. In both traces we find cases for which the change in altitude of the layer with time can be characterized by either a cusp or a steep slope containing an inflection point. Both of these features are consistent with wave steepening. That is, these data provide direct evidence for the connection between wave steepening and the observed jumps in the spectrograms.

The last example of spectrogram data is shown in Fig. 14. The notable characteristics of the spectra are the occasionally extremely narrow widths and numerous jumps. During the time interval when the spectrogram is most discontinuous, between 01:20 and 01:40 UT, there are also many peaks in the SNR and coherence. Based on these data, it would appear that a jump in the Doppler spectrum corresponds approximately to a peak in the SNR and a peak in the coherence. It is difficult to identify whether phase offsets exist between these relationships. For comparison, we note that Chilson et al. (1997b) used FDI data from the SOUSY VHF radar to investigate the 
dynamics of KH billows associated with an upper level jet stream. They found that maxima in the SNR coincided with increases in the radial velocity, increases in the coherence, and minima in the layer altitudes. These authors were lead to conclude that the maxima in SNR and coherence resulted from partial reflections from large-scale stratifications of the refractive index. More data examples will be needed to make definitive connections between occurrences of the spectral jumps and the maxima/minima of the SNR and coherence for PMSE.

\section{Conclusions}

A central problem in understanding the source of PMSE is locating a suitable production mechanism for the electron fluctuations that could be seen by radar. Existing rocket and radar observations suggest that non-turbulent mechanisms must play a role for the generation and/or preservation of PMSE (Lübken et al., 1993, 1998). As summarized in Cho and Röttger (1997), four potential mechanisms are dust-hole scatter, opalescence, charged-dust-diffusive waves, and vertical convergence. We may be detecting one or more of these processes; however, at present insufficient data exist to thoroughly weigh the strengths and weaknesses of any of these theories. It may be necessary to implement more sophisticated radar methods such as coherent radar imaging (CRI) (Palmer et al., 1998) and/or range imaging (RIM) (Palmer et al., 1999) to answer these questions.

Some of the features in our data may be explained by sharp edges on turbulent layers and highly anisotropic scatterers at the edges of turbulent layers (Hocking, 1987; Woodman and Chu, 1989). We have shown that FDI is capable of identifying such layers within PMSE and even providing estimates of their locations and widths. We recognize that the FDI technique suffers from some limitations; chief among these is that the mathematical development of FDI relies on the assumption that at most one scattering layer is present within a given range gate. We note that this assumption can be relaxed when implementing RIM with three or more frequencies. Despite the limitations, we strongly believe that FDI and other frequency-diverse radar techniques will play a significant role in advancing our understanding of PMSE and the atmospheric dynamics that the modulate them.

The horizontally structured layers that we have examined using FDI could have been formed by, among other mechanisms, KH instabilities. It is believed that these instabilities are common near the mesopause and, although never directly observed, there have been several reports in the literature in which evidence has been found to support the belief (Pan and Röttger, 1996; Czechowsky and Rüster, 1997). High-resolution computer simulations are also being developed that can be used to study the impact on KH instabilities on PMSE (Werne and Fritts, 1999; Hill et al., 1999). Although this work is still newly developing, it appears that these simulations offer a powerful tool for investigating the links between PMSE and atmospheric small-scale dynamics.

We have successfully incorporated FDI processing techniques into our investigation of PMSE and wave dynamics. It is believed that upwardly propagating atmospheric waves become steepened near the mesopause where their energy is transferred nonlinearly from the fundamental wave frequency to higher frequency harmonics (Mobbs, 1985; Weinstock, 1986, 1987). Indications of wave steepening have been found in optical images of NLC (Witt, 1962) as well as radar observations of PMSE (Röttger et al., 1990). Our analysis using RTP plots of SNR, spectrograms of Doppler velocity, and estimates of the positions of individual scattering layers is in agreement with the model put forward by Röttger et al. (1990) regarding how steepened waves would manifest in PMSE data.

We add in conclusion that these data suggest that FDI is capable of locating horizontal structures in the plasma within PMSE. This supposition would need to be validated through, e.g., complementary measurements with rocket probes similar to those reported by Lübken et al. (1993, 1998). For such an experiment, it would be advisable to operate the radar used to detect the PMSE in a multiple-receiver and multiple-frequency mode if possible.

Acknowledgements. We appreciate assistance with the configuration and operation of the FDI experiment from Tony van Ayken and Gudmund Wannberg. We thank D. Hooper for his helpful comments during the preparation of this study. We are very grateful for the wind data from the Alomar SOUSY radar provided by the Leibniz-Institute für Atmosphärenphysik in Kühlungsborn and for Peter Hoffmann's assistance in preparing them. We are also grateful for data from the Imaging Riometer for Ionospheric Studies (IRIS) provided by the Communications Research Centre at Lancaster University. P.B.C. was supported by the Environment and Space Research Institute (MRI) in Kiruna. S.K. is financed by the Swedish Natural Science Research Council (NFR) and MRI. The EISCAT Scientific Association is funded by CNRS (France), MPG (Germany), NFR (Sweden), NIPR (Japan), RCN (Norway), PPARC (UK), and SA (Finland). IRIS is funded by the Particle Physics and Astronomy Research Council (PPARC) in collaboration with the Sodankylä Geophysical Observatory.

Topical Editor Mark Lester thanks two Referees for their help in evaluating this paper.

\section{References}

Chilson, P. B., and G. Schmidt, Implementation of frequency domain interferometry at the SOUSY VHF radar: first results, Radio Sci., 31, 263-272, 1996.

Chilson, P. B., P. Czechowsky, J. Klostermeyer, R. Rüster, and G. Schmidt, An investigation of measured temperature profiles and VHF mesosphere summer echoes at midlatitudes, J. Geophys. Res., 102, 23 819-23 828, 1997a.

Chilson, P. B., A. Muschinski, and G. Schmidt, First observations of Kelvin-Helmholtz billows in an upper level jet using VHF frequency domain interferometry, Radio Sci., 32, 1149-1160, 1997b.

Cho, J. Y. N., and J. Röttger, An updated review of polar mesosphere summer echoes: observation, theory, and their 
relationship to noctilucent clouds and subvisible aerosols, J. Geophys. Res., 102, 2001-2020, 1997.

Cho, J. Y. N., W. E. Swartz, M. C. Kelley, and C. A. Miller, CUPRI observations of PMSE during SALVOB of NLC-91: evidence of both partial reflection and turbulent scatter, Geophys. Res. Lett., 20, 2291-2294, 1993.

Czechowsky, P., and R. Rüster, VHF radar observations of turbulent structures in the polar mesopause region, Ann. Geophysicae, 15, 1028-1036, 1997.

Doviak, R., and D. S. Zrnić, Reflection and scatter formula for anisotropically turbulent air, Radio Sci., 19, 325-336, 1984.

Ecklund, W. L., and B. B. Balsley, Long-term observations of the arctic mesopause with the radar at Poker Flat, Alaska, J. Geophys. Res., 86, 7775-7780, 1981.

Franke, S. J., Pulse compression and frequency domain interferometry with a frequency-hopped MST radar, Radio Sci., 25, 565-574, 1990.

Franke, S. J., J. Röttger, and C. La Hoz, Frequency domain interferometry of polar mesosphere summer echoes with the EISCAT VHF radar: a case study, Radio Sci., 27, 417-428, 1992.

Fritts, D. C., S. A. Smith, B. B. Balsley, and C. R. Philbrick, Evidence of gravity wave saturation and local turbulence production in the summer mesosphere and lower thermosphere during the STATE experiment, J. Geophys. Res., 93, 7015-7025, 1988.

Havnes, O., F. Melandso, C. L. Hoz, T. K. Aslaksen, and T. Hartquist, Charged dust in the Earth's mesopause: effects on radar backscatter, Phys. Scripta, 45, 535-544, 1992.

Hill, R. J., D. E. Gibson-Wilde, J. A. Werne, and D. C. Fritts, Turbulence-induced fluctuations in ionization and application to PMSE, Earth Planet. Space, 51, 499-513, 1999.

Hocking, W., Radar studies of small-scale structure in the upper middle atmosphere and lower ionosphere, Adv. Space Res., 7, 327-338, 1987.

Hocking, W. K., and J. Röttger, Studies of polar mesosphere summer echoes over EISCAT uing calibrated signal strengths and statistical parameters, Radio Sci., 32, 1425-1444, 1997.

Hoffmann, P., W. Singer, and J. Bremer, Mean seasonal and diurnal variations of PMSE and winds from 4 years of radar observations at ALOMAR, Geophys. Res. Lett., 26, 1525-1528, 1999.

Kirkwood, S., V. Barabash, P. Chilson, A. Réchou, K. Stebel, P. Espy, G. Witt, and J. Stegman, The 1997 PMSE season - its relation to wind, temperature and water vapour, Geophys. Res. Lett., 25, 1867-1870, 1998.

Kudeki, E., and R. Stitt, Frequency domain interferometry studies of mesospheric layers at Jicamarca, Radio Sci., 25, 575- 590, 1990.

La Hoz, C., J. Röttger, M. Rietveld, G. Wannberg, and S. J. Franke, The status and planned development of EISCAT in mesosphere and D-region experiments, in Handbook for MAP, vol 28, 476-488, SCOSTEP Secr., University of Illinois, Urbana, 1989.

Lübken, F.-J., Thermal structure of the Arctic summer mesopause, J. Geophys. Res., 104, 9135-9150, 1999.

Lübken, F.-J., G. Lehmacher, T. Blix, U.-P. Hoppe, E. Thrane, J. Cho, and W. Swartz, First in-situ observations of neutral and plasma density fluctuations within a PMSE layer, Geophys. Res. Lett., 20, 2311-2314, 1993.

Lübken, F.-J., M. Rapp, T. Blix, and E. Thrane, Microphysical and turbulent measurements of the Schmidt number in the vicinity of polar mesosphere summer echoes, Geophys. Res. Lett., 25, 893-896, 1998.

Miles, J. W., and L. N. Howard, Note on a heterogeneous shear flow, Q. J. R. Meteorol. Soc., 20, 331-336, 1964.

Mobbs, S. D., Propagation of nonlinear internal gravity waves at stratospheric and mesospheric heights. Part iii: the wave shape, Ann. Geophysicae, 3, 599-608, 1985.

Muschinski, A., P. B. Chilson, S. Kern, J. Nielinger, G. Schmidt, and T. Prenosil, First frequency-domain interferometry obser- vations of large-scale vertical motion in the atmosphere, J. Atmos. Sci., 56, 1248-1258, 1999.

Nastrom, G. D., and F. D. Eaton, Turbulence eddy dissipation rates from radar observations at 5-20 km at White Sands Missile Range, NM, J. Geophys. Res., 102, 19 495-19 505, 1997.

Nussbaumer, V., K. H. Fricke, M. Langer, W. Singer, and U. von Zahn, First simultaneous and common volume observations of noctilucent clouds and polar mesosphere summer echoes by lidar and radar, J. Geophys. Res., 101, 19 161-19 167, 1996.

Palmer, R. D., S. Gopalam, T.-Y. Yu, and S. Fukao, Coherent radar imaging using Capon's method, Radio Sci., 33, 1585-1589, 1998.

Palmer, R. D., T.-Y. Yu, and P. B. Chilson, Radio imaging using frequency diversity, Radio Sci., 34, 1485-1496, 1999.

Pan, C. J., and R. Röttger, Structures of polar mesosphere summer echoes observed with the EISCAT VHF in the interferometric mode, in Solar-Teresstrial Energy Program: Proceedings of the Seventh Workshop on Technical and Scientific Aspects of MST Radar, 252-255, SCOSTEP Secretariat, Boulder, CO, 1996.

Reid, I. M., P. Czechowsky, R. Rüster, and G. Schmidt, First VHF radar measurements of mesopause summer echoes at midlatitudes, Geophys. Res. Lett., 16, 135-138, 1989.

Röttiger, J., and C. L. Hoz, Characteristics of polar mesosphere summer echoes (PMSE) observed with the EISCAT 224-MHz radar and possible explanations of their origin, J. Atmos. Terr. Phys., 52, 893-906, 1990.

Röttiger, J., C. L. Hoz, M. C. Kelley, U.-P. Hoppe, and C. Hall, The structure and dynamics of polar mesosphere summer echoes observed with the EISCAT $224 \mathrm{MHz}$ radar, Geophys. Res. Lett., 15, 1353-1356, 1988.

Röttiger, J., C. L. Hoz, S. J. Franke, and C. H. Liu, Steepening of reflectivity structures detected in high-resolution Doppler spectra of polar mesosphere summer echoes (PMSE) observed with the EISCAT 224-MHz radar, J. Atmos. Terr. Phys., 52, 939954, 1990.

Rüster, R., High resolution measurements in the summer polar mesosphere, Geophys. Res. Lett., 24, 1115-1118, 1997.

Rüster, R., P. Czechowsky, P. Hoffmann, and W. Singer, Gravity wave signatures at mesopause heights, Ann. Geophysicae, 14, 1186-1191, 1996.

Singer, W., D. Keuer, P. Hoffmann, P. Czechowsky, and G. Schmidt, The ALOMAR SOUSY radar: technical design and further develoments, in Proc of the 12th ESA Symposium on European Rocket and Balloon Programmes and Related Research, 409-415, 1995.

Stebel, K., V. Barabash, S. Kirkwood, J. Siebert, and K.-H. Fricke, Polar mesosphere summer echoes and noctilucent clouds: Simultaneous and common-volume observations by radar, lidar, and CCD camera, Geophys. Res. Lett., 27, 661-664, 2000.

Thomas, L., I. Astin, and T. Prichard, The characteristics of VHF echoes from the summer mesopause region at mid-latitudes, J. Atmos. Terr. Phys., 54, 969-977, 1992.

von Zahn, U., and J. Bremer, Simultaneous and common-volume observations of noctilucent clouds and polar mesosphere summer echoes, Geophys. Res. Lett., 26, 1521-1524, 1999.

Weinstock, J., Finite amplitude gravity waves: harmonics, advective steepening and saturation, J. Atmos. Sci., 43, 688-704, 1986.

Weinstock, J., The turbulence field generated by a linear gravity wave, J. Atmos. Sci., 44, 410-420, 1987.

Werne, J., and D. C. Fritts, Stratified shear turbulence: Evolution and statistics, Geophys. Res. Lett., 26, 439-442, 1999.

Witt, G., Height, structure and displacements of noctilucent clouds, Tellus, 14, 1-18, 1962.

Woodman, R. F., Spectral moment estimation in MST radars, Radio Sci., 20, 1185-1195, 1985.

Woodman, R. F., and Y.-H. Chu, Aspect sensitivity measurements of VHF backscatter made with the Chung-Li radar: plausible mechanisms, Radio Sci., 24, 113-125, 1989.

Zecha, M., Untersuchung der polaren sommerlichen Mesosphäre mit untershiedlichen Radarverfahren im VHF-Bereich, $\mathrm{PhD}$ Thesis, Universität Rostock, Germany, 1999. 